\title{
Jugular Venous Reflux Is Associated with Perihematomal Edema after Intracerebral Hemorrhage
}

\author{
Hao Feng, ${ }^{1,2,3,4}$ Hongxia Zhang, ${ }^{5}$ Wen He, ${ }^{5}$ Jian Zhou, ${ }^{6}$ and Xingquan Zhao ${ }^{1,2,3,4}$ \\ ${ }^{1}$ Department of Neurology, Beijing Tiantan Hospital, Capital Medical University, Beijing 100050, China \\ ${ }^{2}$ China National Clinical Research Center for Neurological Diseases, Beijing 100050, China \\ ${ }^{3}$ Center of Stroke, Beijing Institute for Brain Disorders, Beijing 100050, China \\ ${ }^{4}$ Beijing Key Laboratory of Translational Medicine for Cerebrovascular Disease, Beijing 100050, China \\ ${ }^{5}$ Department of Diagnostic Ultrasound, Beijing Tiantan Hospital, Capital Medical University, Beijing 100050, China \\ ${ }^{6}$ Department of Radiology, Beijing Tiantan Hospital, Capital Medical University, Beijing 100050, China
}

Correspondence should be addressed to Jian Zhou; zhoujianttyy@163.com and Xingquan Zhao; zxq@vip.163.com

Received 14 November 2016; Revised 1 April 2017; Accepted 11 May 2017; Published 11 June 2017

Academic Editor: Pasquale De Bonis

Copyright (C) 2017 Hao Feng et al. This is an open access article distributed under the Creative Commons Attribution License, which permits unrestricted use, distribution, and reproduction in any medium, provided the original work is properly cited.

\begin{abstract}
The purpose of this study was to determine whether jugular venous reflux (JVR) is associated with perihematomal edema (PHE) in individuals with intracerebral hemorrhage (ICH). Patients with spontaneous supratentorial ICH within $72 \mathrm{~h}$ of symptom onset were enrolled. Baseline brain computed tomography (CT) scan was performed, with a follow-up CT examination at $12 \pm 3$ days after onset. Jugular venous color Doppler ultrasound was performed at $12 \pm 3$ days after onset to examine the JVR status. A total of 65 patients with ICH were enrolled. In logistic regression analysis, absolute PHE volume was significantly associated with JVR (OR, 5.46; 95\% CI, 1.04-28.63; $p=0.044$ ) and baseline hematoma volume (OR, 1.14; 95\% CI, 1.03-1.26; $p=0.009$ ) within $72 \mathrm{~h}$ of onset. It was also correlated with JVR (OR, 15.32; 95\% CI, 2.52-92.99; $p=0.003)$ and baseline hematoma volume (OR, 1.14; 95\% CI, $1.04-1.24 ; p=0.006)$ at $12 \pm 3$ days after onset. In a similar manner, relative PHE volume was significantly associated with JVR (OR, 14.85 ; 95\% CI, 3.28-67.17; $p<0.001$ ) within $72 \mathrm{~h}$ of onset and at $12 \pm 3$ days after onset (OR, 5.87; 95\% CI, 1.94-17.77; $p=0.002$ ). JVR is associated with both absolute and relative PHE volumes after ICH.
\end{abstract}

\section{Introduction}

Intracerebral hemorrhage $(\mathrm{ICH})$ is a neurological disease with mortality rates within 30 days reaching $30-52 \%$ [1-3]. Besides the initial hematoma volume, subsequent growth and other factors such as age, intraventricular blood, and perihematomal edema (PHE) also contribute to poor outcome $[4,5]$. Indeed, PHE is considered an important prognostic factor in patients with ICH [6]. As part of secondary brain damage in ICH, PHE begins to develop within the first $24 \mathrm{~h}$ of onset [7] and grows rapidly in the first three days, peaking about two weeks later [8].

Jugular venous reflux (JVR) is widely found in healthy individuals, with a prevalence of about $20-40 \%$, which increases with age [9]. JVR is characterized by a retrograde flow in the internal jugular veins (IJVs) during Valsalvalike maneuvers (VM) or spontaneously [10]. Previous studies have demonstrated that JVR can retrogradely transmit hypertension into the cerebral venous system and is associated with structural changes in the brain parenchyma of patients with mild cognitive impairment and Alzheimer's disease, increasing vasogenic edema in the brain $[10,11]$. Preliminary data showed that cranial venous outflow abnormalities increase brain edema after arterial ischemic stroke [12]. Based on the above findings, we hypothesized that JVR increases PHE volume after ICH.

To test this hypothesis, this cohort study was carried out with the aim to assess the association of JVR and PHE volume.

\section{Methods}

2.1. Patients. This was a cohort study assessing patients with spontaneous supratentorial intracerebral hemorrhage 
admitted to the Department of Neurology, Beijing Tiantan Hospital, China. The inclusion criteria were (1) age $\geq 18$; (2) admission diagnosis of supratentorial ICH confirmed by brain computed tomography (CT); and (3) baseline CT scan completion within $72 \mathrm{~h}$ of onset. The exclusion criteria were (1) infratentorial hemorrhage; (2) primary intraventricular hemorrhage; (3) subsequent surgery; or (4) any suspected cause of secondary ICH. Data were prospectively collected and retrospectively analyzed. This study was approved by the Ethics Committee of Beijing Tiantan Hospital affiliated to the Capital Medical University of China, in compliance with the Declaration of Helsinki. All patients or their legal representatives provided signed informed consent.

2.2. Collection of Patient Baseline Characteristics. Eligible patients were assessed for demographics data, clinical data, and hematoma and PHE volumes. Blood samples were collected from each patient on the day of hospital admission for the measurement of blood glucose (GLU), low-density lipoprotein (LDL) levels, and international normalized ratio (INR). Clinical and neurological evaluations were performed to collect data, including first blood pressure measurement after onset, as well as history of smoking, hemorrhagic and ischemic stroke, diabetes mellitus, hypertension, coronary heart disease, and blood diseases (including coagulopathy and leukemia). History of smoking was defined as currently smoking or past smoking (i.e., no smoking for the past 5 years). Arterial hypertension was defined as systolic blood pressure $\geq 140 \mathrm{mmHg}$ and/or diastolic blood pressure $\geq 90 \mathrm{mmHg}$, or self-reported current treatment for arterial hypertension with antihypertensive medications. On the admission day, the patients were assessed for stroke severity, consciousness state, and the level of handicap according to the National Institute of Health Stroke Scale (NIHSS), Glasgow Coma Scale (GCS), and modified Rankin Scale $(\mathrm{mRS})$, respectively.

2.3. Color Doppler Ultrasound. Color Doppler ultrasound was carried out on GE Logiq9 (General Electric, Fairfield, USA), with the patient lying flat in the supine position and the head straight, using a 4-7 MHz linear transducer after a 10 min of quiet rest. All examinations were performed by an experienced neurosonographer blinded to the patient's clinical data. Sufficient ultrasound gel was applied and appropriate care was given to avoid compression on the neck veins during examination. Bilateral internal jugular veins (IJVs) were examined initially through longitudinal and cross-sectional views from the proximal part of the neck base rostrally to the distal part at the submandibular level to detect spontaneous JVR. The color box was adjusted to include the entire IJV lumen. When retrograde color appeared in the lumen center, the retrograde flow was confirmed by Doppler spectrum. JVR was defined as the retrograde flow $>0$ seconds detected at the distal part of the IJV (above the inferior jugular bulb) (Figure 1) [11, 13, 14]. Patients with JVR on neither side were classified as JVR-negative, while those with JVR detected on either or both sides simultaneously were considered to be JVR-positive. We also detected structural abnormalities of the

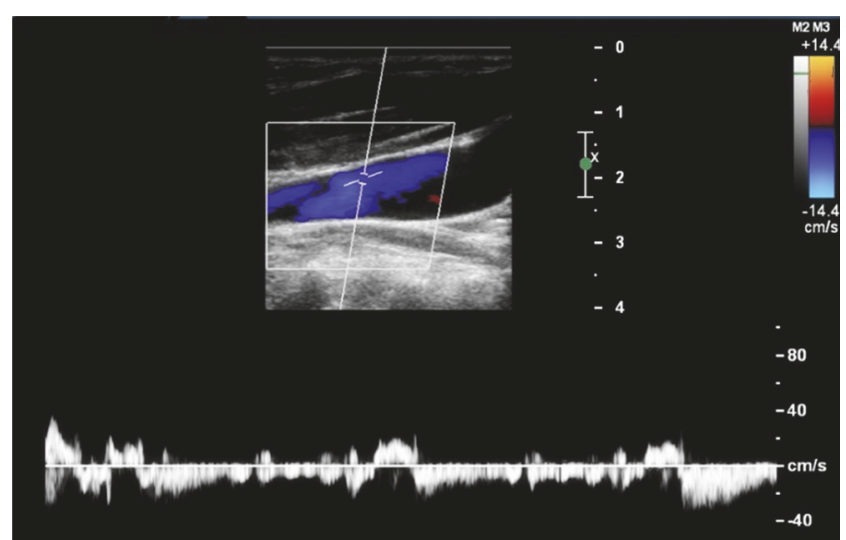

Figure 1: Retrograde flow detected in the Doppler spectrum spontaneously is considered jugular venous reflux (JVR).

IJVs as potential causes of JVR $[15,16]$. All structural abnormalities were classified into two subcategories: intraluminal structural (web, flap, septa, membrane, and malformed valve) and extraluminal structural (stenosis and annulus) [15].

2.4. Computed Tomography. Baseline noncontrast computed tomography (NCCT) was performed within $72 \mathrm{~h}$ of onset and repeated at $12 \pm 3$ days, on a CT scanner (General Electric), with $512 \times 512$ matrix, FOV of $15 \mathrm{~cm}$, and slice thickness of 9 or $10 \mathrm{~mm}$ (supratentorial) and 4.5 or $5 \mathrm{~mm}$ (infratentorial). A roundish or ellipsoid hematoma with smooth margin was classified as regular: one with a pleomorphic contour and several adjacent but separated hematomas, and multicentric hematomas were classified as irregular. Intracranial hematoma (excluding ventricular hemorrhage) and $\mathrm{PHE}$ volumes were independently measured by two trained neurologists blinded to clinical data. Images were processed off-line with the Picture Archiving and Communications Systems (PACS). The examiner manually drew regions of interest (ROIs) by tracing the hyperdense area (hematoma) and hypodense region surrounding the hematoma (PHE) throughout the lesion [17]. Threshold ranges for hematomas and edemas were 44-100 and 5-33 Hounsfield units, respectively. Hematoma volume for each slice was then calculated by multiplying the hyperdense area by slice thickness. Hematoma volumes from all slices were added to obtain total hematoma volumes. Similarly, total lesion volumes (hyperdense + hypodense) were calculated. Absolute PHE volume was measured by subtracting the hematoma volume from total lesion volume. Relative PHE was calculated by dividing the absolute PHE volume by that of hematoma. When PHE was too small to measure, a value of zero was assigned for both absolute and relative PHE volumes [17]. The subjects were grouped based on average absolute and relative PHE volumes, into the large-volume (greater than average PHE volume) and small-volume (less than average PHE volume) groups, respectively.

2.5. Statistical Analysis. SPSS for Windows, version 16.0 (IBM, Armonk, NY, USA), was used for all statistical analyses. 
TABLE 1: Baseline characteristics and risk factor profiles of patients with ICH.

\begin{tabular}{|c|c|c|c|}
\hline & JVR negative & JVR positive & $p$ value \\
\hline Number of subjects, $n$ & 36 & 29 & NA \\
\hline Age in years, mean $(S D)$ & $52.9(12.2)$ & $55.1(9.3)$ & 0.422 \\
\hline Male gender, $n(\%)$ & $27(75.0)$ & $24(82.8)$ & 0.449 \\
\hline History of smoking, $n(\%)$ & $14(38.9)$ & $13(44.8)$ & 0.629 \\
\hline History of ICH, $n(\%)$ & $0(0.0)$ & $4(13.8)$ & 0.075 \\
\hline History of IS, $n(\%)$ & $4(11.1)$ & $3(10.3)$ & NA \\
\hline History of CAD, $n(\%)$ & $2(5.6)$ & $2(6.9)$ & NA \\
\hline History of hypertension, $n(\%)$ & $29(80.6)$ & $26(89.7)$ & 0.506 \\
\hline Irregular shape of hematoma, $n(\%)$ & $5(13.9)$ & $9(36 \%)$ & 0.095 \\
\hline Admission GCS, median (IQR) & $15(13-15)$ & $14(12-15)$ & 0.264 \\
\hline Admission mRS, median (IQR) & $4(2-4)$ & $4(2-5)$ & 0.308 \\
\hline Admission NIHSS, mean (SD) & $7.8(5.1)$ & $8.4(5.4)$ & 0.614 \\
\hline $\mathrm{LDL}(\mathrm{mmol} / \mathrm{L})$, mean $(\mathrm{SD})$ & $3.1(0.9)$ & $2.8(0.5)$ & 0.203 \\
\hline GLU (mmol/L), mean (SD) & $5.3(1.4)$ & $5.0(1.3)$ & 0.509 \\
\hline INR, mean $(S D)$ & $1.00(0.06)$ & $1.01(0.04)$ & 0.214 \\
\hline First SBP $(\mathrm{mmHg})$, mean $(\mathrm{SD})^{\dagger}$ & $171(29)$ & $162(24)$ & 0.184 \\
\hline First DBP $(\mathrm{mmHg})$, mean $(\mathrm{SD})^{\dagger}$ & $99(16)$ & $100(15)$ & 0.771 \\
\hline Baseline hematoma volume (ml), mean (SD) & $19.52(12.49)$ & $20.29(10.85)$ & 0.794 \\
\hline sIVH, $n(\%)$ & $6(16.7)$ & $5(17.2)$ & 1.000 \\
\hline $\begin{array}{l}\text { Hematoma location in basal ganglia or thalamus, } n \\
(\%)\end{array}$ & $31(86.1)$ & $26(90.0)$ & 0.958 \\
\hline Intraluminal structural abnormality, $n(\%)$ & $2(5.6)$ & $5(17.2)$ & 0.268 \\
\hline Malformed valve & $2(5.6)$ & $5(17.2)$ & - \\
\hline Flap & 0 & 0 & \\
\hline Web & 0 & 0 & \\
\hline Membrane & 0 & 0 & \\
\hline Septum & 0 & 0 & \\
\hline Extraluminal structural abnormality, $n(\%)$ & 0 & 0 & - \\
\hline
\end{tabular}

NA, not applicable; ICH, intracerebral hemorrhage; IS, ischemic stroke; CAD, coronary artery disease; GCS, Glasgow Coma Scale; mRS, modified Rankin Scale; NIHSS, National Institutes of Health Stroke Scale; LDL, low density lipoprotein; GLU, blood glucose; INR, international normalized ratio; SBP, systolic blood pressure; DBP, diastolic blood pressure; sIVH, secondary intraventricular hemorrhage; IQR, interquartile range; SD, standard deviation; ${ }^{\dagger}$ first blood pressure measured after symptom observation.

Demographic and clinical data of patients (with or without JVR) were assessed by Student's $t$-test or Wilcoxon rank-sum test for continuous variables. The chi-square test or Fisher's exact test was used to compare categorical variables. Multivariate analysis was performed using a logistic regression model to screen risk factors for large PHE. Variables with $p<$ 0.20 were entered in the multivariate analysis, and odds ratios (ORs) and 95\% confidence intervals (CIs) were calculated. Two-sided $p<0.05$ was considered statistically significant.

\section{Results}

3.1. Patient Baseline Characteristics. A total of 65 patients were enrolled in this study. Among them, 11 (16.9\%) and 7 (10.8\%) subjects had right- and left-sided JVR, respectively; 11 patients (16.9\%) had bilateral JVR, while 36 subjects (55.4\%) showed no JVR. Among 29 JVR-positive patients, there were
10 subjects who had retrograde flow in their IJVs with a duration $<0.5 \mathrm{~s}(0.11-0.49 \mathrm{~s})$. The patients with right-sided, left-sided, and bilateral JVR were assigned to the JVR-positive group; those with no JVR were classified into the JVRnegative group.

No extraluminal structural abnormality was found in any subject. In the JVR-negative group, one patient had a dysfunctional valve in the left IJV and one patient had single leaflet valve in the right IJV. Among patients with left-sided JVR, two had single leaflet valve in the left IJV and one had single leaflet valve in the right IJV. Among patients with bilateral JVR, one had single leaflet valve in the left IJV and one had dysfunctional valve in the right IJV. No web, flap, septa, or membrane was observed. The baseline characteristics of the patients are shown in Table 1. No statistically significant differences were found in demographic and baseline clinical parameters between the two groups. 
TABLE 2: PHE volumes in JVR-negative and JVR-positive groups.

\begin{tabular}{|c|c|c|c|}
\hline & JVR negative & JVR positive & $p$ value \\
\hline \multicolumn{4}{|l|}{ Within 72 hours } \\
\hline Absolute PHE volume (ml), mean (SD) & $14.69(12.80)$ & $23.15(14.43)$ & 0.015 \\
\hline Relative PHE volume, mean (SD) & $0.74(0.41)$ & $1.28(0.65)$ & $<0.001$ \\
\hline \multicolumn{4}{|l|}{ At $12 \pm 3$ days } \\
\hline Absolute PHE volume (ml), mean (SD) & $30.67(23.63)$ & $55.56(30.34)$ & $<0.001$ \\
\hline Relative PHE volume, mean (SD) & $2.93(2.23)$ & $6.83(4.32)$ & $<0.001$ \\
\hline
\end{tabular}

PHE, perihematomal edema; SD, standard deviation.

TABLE 3: Numbers of subjects classified based on average PHE volume.

\begin{tabular}{|c|c|c|c|}
\hline & JVR negative & JVR positive & $p$ value \\
\hline Baseline absolute $\mathrm{PHE}^{\dagger}$ & & & 0.041 \\
\hline Large-volume group, $n(\%)$ & $12(41.4)$ & $1(58.6)$ & \\
\hline Small-volume group, $n(\%)$ & $24(66.7)$ & $12(33.3)$ & \\
\hline Baseline relative $\mathrm{PHE}^{\ddagger}$ & & & $<0.001$ \\
\hline Large-volume group, $n(\%)$ & $6(24.0)$ & $19(76.0)$ & \\
\hline Small-volume group, $n(\%)$ & $30(75.0)$ & $10(25.0)$ & \\
\hline $12 \pm 3$ days' absolute $\mathrm{PHE}^{\S}$ & & & 0.001 \\
\hline Large-volume group, $n(\%)$ & $8(30.8)$ & $18(69.2)$ & \\
\hline Small-volume group, $n(\%)$ & $28(71.8)$ & $11(28.2)$ & \\
\hline $12 \pm 3$ days' relative $\mathrm{PHE}^{/ /}$ & & & 0.001 \\
\hline Large-volume group, $n(\%)$ & $7(29.2)$ & $17(70.8)$ & \\
\hline Small-volume group, $n(\%)$ & $29(70.7)$ & $12(29.3)$ & \\
\hline
\end{tabular}

PHE, perihematomal edema; ${ }^{\dagger}$ patients classified according to average absolute PHE volume $(18.47 \mathrm{ml})$ within $72 \mathrm{~h}$ of onset; ${ }^{\ddagger}$ patients classified according to average relative PHE volume (0.98) within $72 \mathrm{~h}$ of onset; ${ }^{\$}$ patients classified according to average absolute PHE volume (41.77 ml) at $12 \pm 3$ days after onset; "patients classified according to average relative PHE volume (4.67) at $12 \pm 3$ days after onset.

3.2. JVR Increases PHE Volumes in ICH Patients. To characterize the relationship between JVR and PHE in ICH patients, PHE volumes were compared between the two groups within $72 \mathrm{~h}$ of onset and at $12 \pm 3$ days after onset. Interestingly, both absolute and relative PHE volumes in the JVR-positive group were significantly higher than those of the JVR-negative group (Table 2).

3.3. Association of PHE and JVR. To further explore the relationship between PHE and JVR, the subjects were further grouped based on average absolute and relative $\mathrm{PHE}$ volumes, into the large-volume (exceeding average PHE volume) and small-volume (less than average PHE volume) groups (Table 3). Interestingly, the JVR prevalence in the largevolume group was significantly higher than that in the smallvolume group.

3.4. Association of PHE Volume with Clinicopathologic Parameters. Logistic regression analysis was carried out for each of the four conditions shown in Table 3. In univariate analyses, to avoid missing relevant variables, an association was considered statistically significant at $p<0.20$. Absolute PHE volume within $72 \mathrm{~h}$ of onset was significantly associated with JVR $(p<0.05)$, sex $(p=0.173)$, smoking history $(p=$
0.135), hematoma shape $(p=0.095)$, NIHSS on admission $(p$ $<0.001)$, mRS on admission $(p<0.05)$, GCS on admission $(p$ $<0.05)$, baseline hematoma volume $(p<0.001)$, first systolic blood pressure $(p=0.189)$, and first diastolic blood pressure ( $p$ $<0.05)$. Meanwhile, relative PHE volume within $72 \mathrm{~h}$ of onset was significantly associated with JVR $(p<0.001)$, hematoma shape $(p<0.05)$, NIHSS on admission $(p=0.171)$, GLU $(p=$ $0.128)$, baseline hematoma volume $(p<0.001)$, first systolic blood pressure $(p=0.189)$, and first diastolic blood pressure $(p<0.05)$. Furthermore, absolute PHE volume at $12 \pm 3$ days after onset was significantly associated with JVR ( $p=$ $0.001)$, sex $(p=0.005)$, hematoma shape $(p<0.05)$, NIHSS on admission $(p=0.008)$, mRS on admission $(p=0.071)$, GCS on admission $(p=0.041)$, baseline hematoma volume $(p<0.001)$, and LDL $(p=0.051)$. Moreover, relative PHE at $12 \pm 3$ days after onset was significantly associated with JVR $(p=0.001)$.

In multivariate logistic regression analysis, PHE volume remained significantly associated with JVR in all four conditions. JVR and baseline hematoma volume showed significant associations with absolute and relative PHE volumes, within 3 and $12 \pm 3$ days of onset, respectively, as shown in Table 4 .

3.5. Analysis of the Threshold for JVR. The patients were regrouped according to the duration of JVR. Patients with JVR below $0.5 \mathrm{~s}$ were considered JVR-negative because JVR 
TABLE 4: Odd ratios in multivariate logistic regression analysis.

\begin{tabular}{lcc}
\hline & OR value (95\% CI) & $p$ value \\
\hline Based on 72 h absolute PHE $^{\dagger}$ & & \\
JVR & $5.46(1.04-28.63)$ & 0.044 \\
$\quad$ Baseline hematoma volume & $1.14(1.03-1.26)$ & 0.009 \\
$\begin{array}{l}\text { Based on } 72 \mathrm{~h} \text { relative PHE } \\
\quad \text { JVR }\end{array}$ & $14.85(3.28-67.17)$ & $<0.001$ \\
$\begin{array}{l}\text { Based on absolute PHE at } 12 \pm 3 \\
\text { days }\end{array}$ & & \\
$\quad$ JVR & $15.32(2.52-92.99)$ & 0.003 \\
$\quad \begin{array}{l}\text { Baseline hematoma volume } \\
\text { Based on relative PHE at } 12 \pm 3 \\
\text { days }\end{array}$ & $1.14(1.04-1.24)$ & 0.006 \\
$\quad$ JVR & & \\
\hline
\end{tabular}

PHE, perihematomal edema; CI, confidence interval; OR, odds ratio; ${ }^{\dagger}$ patients classified according to average absolute PHE volume within $72 \mathrm{~h}$ of onset; ${ }^{\ddagger}$ patients classified according to average relative PHE volume within $72 \mathrm{~h}$ of onset; ${ }^{\$}$ patients classified according to average absolute PHE volume at $12 \pm 3$ days after onset; " patients classified according to average relative PHE volume at $12 \pm 3$ days after onset.

below $0.5 \mathrm{~s}$ was considered normal in some previous studies $[10,14,18-24]$. In the multivariate analysis, the OR of JVR was 7.35 (95\% CI: $1.15-47.20 ; p=0.035$ ) for $72 \mathrm{~h}$ absolute PHE; OR $=7.15$ (95\% CI: $1.64-31.15 ; p=0.009)$ for $72 \mathrm{~h}$ relative $\mathrm{PHE} ; \mathrm{OR}$ $=17.46$ (95\% CI: 3.21-94.96; $p=0.001)$ for absolute PHE at 12 \pm 3 days; and OR $=6.89$ (95\% CI: $2.12-22.46 ; p=0.001$ ) for relative $\mathrm{PHE}$ at $12 \pm 3$ days. These results are similar to those obtained when using JVR $>0 \mathrm{~s}$ as the threshold for JVR.

\section{Discussion}

This study demonstrated that PHE volumes were significantly higher in ICH patients with JVR compared with the JVRnegative group. In addition, JVR was associated with both absolute and relative PHE volumes in these patients after adjusting for age, sex, first blood pressure measurement after symptom detection, baseline hematoma volume, hematoma shape, GLU, LDL, NIHSS, GCS, and mRS on admission.

It is admitted that hematoma volume is the main factor that determines the volume of PHE $[8,25]$. In this study, baseline hematoma volumes in the JVR- positive group were similar to those of JVR-negative patients. Additionally, differences in other clinical factors that may affect PHE volume (such as age, gender, GLU, NIHSS, GCS, and history of smoking, hypertension, and stroke) were not statistically significant between the two groups. These findings suggested that JVR status, as the only difference between the two patient groups, may play an important role in the formation of PHE. This is the first study demonstrating that JVR is associated with PHE after ICH.

Venous flow from the superficial and deep venous system drains into the transverse sinus, then into the sigmoid sinus, and finally into IJV; indeed, IJVs collect most of the cerebral venous blood, especially in the supine position [19, 26, 27].
Therefore, it is quite plausible that hemodynamic changes in IJVs might alter cerebral venous drainage.

Previous studies have shown that JVR in the jugular venous system can cause retrograde hypertension, which can be transmitted to the cerebral venous system $[10,24$, $28,29]$. As shown above, JVR was detected in patients at rest, indicating the retrograde venous pressure may be sustained or repetitive. Such sustained or long-term repetitive retrogradely transmitted venous pressure may subsequently lead to cerebral venous hypertension. As a result, cerebral venules and capillaries dilate [18] and blood brain barrier (BBB) permeability increases, causing large plasma molecules to leak into the brain tissue [10]. One such molecule is endothelin-1 (ET-1), a potent vasoconstrictor peptide derived from vascular endothelial cells, which was shown to synergize with JVR in causing cough syncope/presyncope [14]. The molecules leaking through the $\mathrm{BBB}$ subsequently increase osmotic pressure in the brain tissue [10]. These changes cause water molecules to move from blood vessels to the brain tissue, thereby promoting vasogenic edema formation [30]. Retrograde hypertension in the cerebral venous system, on the other hand, can obstruct cerebrospinal fluid (CSF) circulation and increase intracranial pressure (ICP) [14]. In patients with $\mathrm{ICH}$, the hematoma itself can lead to elevated ICP, which may be aggravated by JRV; the enhanced ICP subsequently reduces cerebral blood flow $(\mathrm{CBF})$ and cerebral perfusion pressure (CPP) [14]. Evidence has shown a decreased perfusion area around the hematoma after ICH; meanwhile, continuous $\mathrm{CBF}$ and $\mathrm{CPP}$ reduction results in an ischemic area around the hematoma, in which $\mathrm{BBB}$ is destroyed alongside increased vasogenic edema $[31,32]$.

In this study, JVR was detected before ICH. It remains unclear why patients with JVR seemed to be normal before ICH. The intracranial venous system contains about $70 \%$ of blood in the cerebral circulation, and ICP can be regulated effectively by the fluctuation of venous blood volume under normal conditions [20,33-39]. With an elevated ICP, however, ICP regulation becomes ineffective so that the abnormal venous outflow contributes to increased venous congestion in patients with high ICP $[20,39]$. A study of ischemic stroke reported in 2009 also confirmed that previous intracranial venous outflow hypoplasia or occlusion may induce early fatal edema after large middle cerebral artery infarction [12].

Most previous studies defined JVR as JVR > $0.5 \mathrm{~s}[10,14$, 18-24], while the present study used any JVR (or JVR $>0 \mathrm{~s}$ ) as the definition for JVR. The exact threshold value for JVR still remains to be clearly defined, but the present study showed that using either $>0 \mathrm{~s}$ or $>0.5 \mathrm{~s}$ resulted in similar results in the multivariate analysis.

JVR is a form of functional abnormality of IJV and is usually observed in conjunction with chronic cerebrospinal venous insufficiency (CCSVI) and internal jugular valve insufficiency (IJVI) [21-23, 40, 41]. The origin of this phenomenon could be the congenital impairment of an IJV valve such as the absence of an IJV valve and acquired dysfunction of an IJV valve, which may be associated with tricuspid valve regurgitation and primary pulmonary hypertension $[42,43]$. 
Tricuspid valve regurgitation and primary pulmonary hypertension could cause elevated central venous pressure leading to damaged IJV valve. Dolic et al. [16] studied 240 healthy individuals and found that the presence of heart disease (especially heart murmurs), obesity, and cigarette smoking were associated with an increased prevalence of intraluminal structural abnormalities such as malformed valve. They inferred that JVR was a secondary effect of intraluminal structural abnormalities [16]. In the present study, there was only a trend of higher percentage of malformed valve in JVR-positive subjects (17.2\%) compared with JVR-negative subjects $(5.6 \%)(p=0.268)$. Other possible cause of JVR should be further explored in future studies.

There are two forms of JVR:VM-induced and spontaneous types [44]. In previous studies, VM was performed by forcible expiration and patients were asked to maintain a Valsalva pressure of $40 \mathrm{mmHg}$ for at least $10 \mathrm{~s}[10,14]$. However, in the present study, most patients with ICH were not able to perform VM and we only detected spontaneous JVR.

Inevitably, this study had some limitations. (1) We focused solely on JVR, a hemodynamic change in the internal jugular vein, without considering other intracranial venous abnormalities. It is unknown whether such venous abnormalities participated in the formation of perihematomal edema in this study. Thus, future research involving a comprehensive evaluation of intracranial and extracranial venous system is strongly needed. (2) The relatively small sample size of this study should be mentioned. (3) For patient safety, only subjects from the general wards in our institution were enrolled, whose baseline hematoma volumes are usually relatively small. Patients in neural intensive care unit with larger hematoma volumes should be also enrolled in future studies under closer monitoring.

\section{Conclusions}

The current findings demonstrated that JVR is strongly associated with PHE volume after ICH. Although the exact mechanism remains unclear, it is plausible that JVR retrogradely transmits venous hypertension into the brain.

\section{Disclosure}

The funders had no role in study design, data collection and analysis, decision to publish, manuscript, or preparation.

\section{Conflicts of Interest}

All authors declared that they have no conflicts of interest.

\section{Acknowledgments}

This study was supported by the Capital Clinical Health Research (Z131107002213009), Beijing Medical High Level Academic Leader (2014-2-010, 2015-2017, Xingquan Zhao), and Beijing Municipal Science \& Technology Commission (Z161100002616008).

\section{References}

[1] G. Xi, R. F. Keep, and J. T. Hoff, "Mechanisms of brain injury after intracerebral haemorrhage," Lancet Neurology, vol. 5, no. 1, pp. 53-63, 2006.

[2] M. Okauchi, G. Xi, R. F. Keep, and Y. Hua, "Tissue-type transglutaminase and the effects of cystamine on intracerebral hemorrhage-induced brain edema and neurological deficits," Brain Research, vol. 1249, pp. 229-236, 2009.

[3] J. Broderick, S. Connolly, E. Feldmann et al., "Guidelines for the management of spontaneous intracerebral hemorrhage in adults: 2007 Update. Guideline from the American Heart Association/American Stroke Association Stroke Council, high blood pressure research council, and the quality of care and outcomes in research interdisciplinary working group," Stroke, vol. 38, no. 6, pp. 2001-2023, 2007.

[4] W. Sun, W. Sun, W. Pan et al., "Predictors of late neurological deterioration after spontaneous intracerebral hemorrhage," Neurocritical Care, vol. 19, no. 3, pp. 299-305, 2013.

[5] S. B. Murthy, Y. Moradiya, J. Dawson, K. R. Lees, D. F. Hanley, and W. C. Ziai, "Perihematomal edema and functional outcomes in intracerebral hemorrhage: influence of hematoma volume and location," Stroke, vol. 46, no. 11, pp. 3088-3092, 2015.

[6] B. Volbers, S. Herrmann, W. Willfarth et al., "Impact of Hypothermia Initiation and Duration on Perihemorrhagic Edema Evolution after Intracerebral Hemorrhage," Stroke, vol. 47, no. 9, pp. 2249-2255, 2016.

[7] J. Yang, H. Arima, G. Wu et al., "Prognostic Significance of Perihematomal Edema in Acute Intracerebral Hemorrhage: Pooled Analysis from the Intensive Blood Pressure Reduction in Acute Cerebral Hemorrhage Trial Studies," Stroke, vol. 46, no. 4, pp. 1009-1013, 2015.

[8] H. Arima, J. G. Wang, Y. Huang et al., "Significance of perihematomal edema in acute intracerebral hemorrhage: the INTERACT trial," Neurology, vol. 73, no. 23, pp. 1963-1968, 2009.

[9] C.-P. Chung, Y.-J. Lin, A.-C. Chao et al., "Jugular venous hemodynamic changes with aging," Ultrasound in Medicine and Biology, vol. 36, no. 11, pp. 1776-1782, 2010.

[10] C. Beggs, C.-P. Chung, N. Bergsland et al., "Jugular venous reflux and brain parenchyma volumes in elderly patients with mild cognitive impairment and Alzheimer's disease," BMC Neurology, vol. 13, article 157, 2013.

[11] C.-P. Chung, A.-C. Chao, H.-Y. Hsu, S.-J. Lin, and H.-H. $\mathrm{Hu}$, "Decreased jugular venous distensibility in migraine," Ultrasound in Medicine and Biology, vol. 36, no. 1, pp. 11-16, 2010.

[12] W. Yu, J. Rives, B. Welch, J. White, E. Stehel, and D. Samson, "Hypoplasia or occlusion of the ipsilateral cranial venous drainage is associated with early fatal edema of middle cerebral artery infarction," Stroke, vol. 40, no. 12, pp. 3736-3739, 2009.

[13] C. Baracchini, S. Tonello, F. Farina et al., "Jugular veins in transient global amnesia: Innocent bystanders," Stroke, vol. 43, no. 9, pp. 2289-2292, 2012.

[14] C.-P. Chung, C.-Y. Cheng, R. Zivadinov et al., "Jugular venous reflux and plasma endothelin-1 are associated with cough syncope: a case control pilot study," BMC Neurology, vol. 13, article 9, 2013.

[15] K. Dolic, K. Marr, V. Valnarov et al., "Intra- and extraluminal structural and functional venous anomalies in multiple sclerosis, as evidenced by 2 noninvasive imaging techniques," American Journal of Neuroradiology, vol. 33, no. 1, pp. 16-23, 2012. 
[16] K. Dolic, B. Weinstock-Guttman, K. Marr et al., "Heart disease, overweight, and cigarette smoking are associated with increased prevalence of extra-cranial venous abnormalities," Neurological Research, vol. 34, no. 8, pp. 819-827, 2012.

[17] Y. Zhu, J.-L. Wang, Z.-Y. He, F. Jin, and L. Tang, "Association of altered serum micro RNAs with perihematomal edema after acute intracerebral hemorrhage," PLoS ONE, vol. 10, no. 7, Article ID e0133783, 2015.

[18] C.-P. Chung, H.-Y. Hsu, A.-C. Chao, C.-Y. Cheng, S.-J. Lin, and H.-H. Hu, "Jugular venous reflux affects ocular venous system in transient monocular blindness," Cerebrovascular Diseases, vol. 29, no. 2, pp. 122-129, 2010.

[19] C.-P. Chung and H.-H. Hu, "Pathogenesis of leukoaraiosis: Role of jugular venous reflux," Medical Hypotheses, vol. 75, no. 1, pp. 85-90, 2010.

[20] H.-Y. Hsu, A.-C. Chao, Y.-Y. Chen et al., "Reflux of jugular and retrobulbar venous flow in transient monocular blindness," Annals of Neurology, vol. 63, no. 2, pp. 247-253, 2008.

[21] P. Lochner, M. Nedelmann, M. Kaps, and E. Stolz, "Jugular valve incompetence in transient global amnesia. A problem revisited," Journal of Neuroimaging, vol. 24, no. 5, pp. 479-483, 2014.

[22] M. Nedelmann, B. M. Eicke, and M. Dieterich, "Functional and morphological criteria of internal jugular valve insufficiency as assesed by ultrasound," Journal of Neuroimaging, vol. 15, no. 1 , pp. 70-75, 2005.

[23] M. Nedelmann, M. Kaps, and W. Mueller-Forell, "Venous obstruction and jugular valve insufficiency in idiopathic intracranial hypertension," Journal of Neurology, vol. 256, no. 6, pp. 964-969, 2009.

[24] C.-P. Chung, P.-N. Wang, Y.-H. Wu et al. et al., "More severe white matter changes in the elderly with jugular venous reflux," Annals of Neurology, vol. 69, no. 3, pp. 553-559, March 2011.

[25] C. Venkatasubramanian, M. Mlynash, A. Finley-Caulfield et al., "Natural history of perihematomal edema after intracerebral hemorrhage measured by serial magnetic resonance imaging," Stroke, vol. 42, no. 1, pp. 73-80, 2011.

[26] D. San Millan, P. Ruiz, D. A. Rufenacht, J. Delavelle, F. Henry, and J. H. Fasel, "The craniocervical venous system in relation to cerebral venous drainage," American journal of neuroradiology, vol. 23, pp. 1500-1508, 2002.

[27] J. M. Valdueza, T. von Münster, O. Hoffman, S. Schreiber, and K. M. Einhäupl, "Postural dependency of the cerebral venous outflow," The Lancet, vol. 355, no. 9199, pp. 200-201, 2000.

[28] S. Marcotti, L. Marchetti, P. Cecconi et al. et al., "An anatomybased lumped parameter model of cerebrospinal venous circulation: can an extracranial anatomical change impact intracranial hemodynamics?" BMC Neurology, vol. 15, p. 95, 2015.

[29] E. F. Toro, L. O. Müller, M. Cristini, E. Menegatti, and P. Zamboni, "Impact of jugular vein valve function on cerebral venous haemodynamics," Current Neurovascular Research, vol. 12, no. 4, pp. 384-397, 2015.

[30] S.-W. Jia, X.-Y. Liu, S. C. Wang, and Y.-F. Wang, "Vasopressin hypersecretion-associated brain edema formation in ischemic stroke: Underlying mechanisms," Journal of Stroke and Cerebrovascular Diseases, vol. 25, no. 6, pp. 1289-1300, 2016.

[31] J.-M. Olivot, M. Mlynash, J. T. Kleinman et al., "MRI profile of the perihematomal region in acute intracerebral hemorrhage," Stroke, vol. 41, no. 11, pp. 2681-2683, 2010.

[32] X. Zhao, Y. Wang, C. Wang, S. Li, Y. Wang, and Z. Yang, "Quantitative evaluation for secondary injury to perihematoma of hypertensive cerebral hemorrhage by functional MR and correlation analysis with ischemic factors," Neurological Research, vol. 28, no. 1, pp. 66-70, 2006.

[33] C. C. Y. Pang, "Autonomic control of the venous system in health and disease: Effects of drugs," Pharmacology and Therapeutics, vol. 90, no. 2-3, pp. 179-230, 2001.

[34] A. Marmarou, K. Shulman, and J. LaMorgese, "Compartmental analysis of compliance and outflow resistance of the cerebrospinal fluid system," Journal of Neurosurgery, vol. 43, no. 5, pp. 523-534, 1975.

[35] B. Schaller, "Physiology of cerebral venous blood flow: from experimental data in animals to normal function in humans," Brain Research Reviews, vol. 46, no. 3, pp. 243-260, 2004.

[36] J. F. Meder, J. Chiras, J. Roland, P. Guinet, S. Bracard, and F. Bargy, "Venous territories of the brain," Journal of Neuroradiology, vol. 21, no. 2, pp. 118-133, 1994.

[37] H. H. Schmidek, L. M. Auer, and J. P. Kapp, “The cerebral venous system," Neurosurgery, vol. 17, no. 4, pp. 663-678, 1985.

[38] Y. Suzuki, H. Ikeda, M. Shimadu, Y. Ikeda, and K. Matsumoto, "Variations of the basal vein: Identification using threedimensional CT angiography," American Journal of Neuroradiology, vol. 22, pp. 670-676, 2001.

[39] Z. Si, L. Luan, D. Kong et al., "MRI-based investigation on outflow segment of cerebral venous system under increased ICP condition," Eur J Med Res, vol. 13, pp. 121-126, 2008.

[40] P. Zamboni, R. Galeotti, E. Menegatti et al., "Chronic cerebrospinal venous insufficiency in patients with multiple sclerosis," Journal of Neurology, Neurosurgery and Psychiatry, vol. 80, no. 4, pp. 392-399, 2009.

[41] I. Domitrz, G. Styczynski, J. Wilczko et al., "Internal jugular vein valve insufficiency is not increased in migraine: an ultrasound study in migraine patients and control participants," The Journal of Headache and Pain, vol. 14, p. 78, 2013.

[42] J. Fisher, F. Vaghaiwalla, J. Tsitlik et al., "Determinants and clinical significance of jugular venous valve competence," Circulation, vol. 65, no. 1 I, pp. 188-196, 1982.

[43] L. P. Dresser and W. M. McKinney, "Anatomic and pathophysiologic studies of the human internal jugular valve," The American Journal of Surgery, vol. 154, no. 2, pp. 220-224, 1987.

[44] R. Zivadinov and C.-P. Chung, "Potential involvement of the extracranial venous system in central nervous system disorders and aging," BMC Medicine, vol. 11, article 260, 2013. 


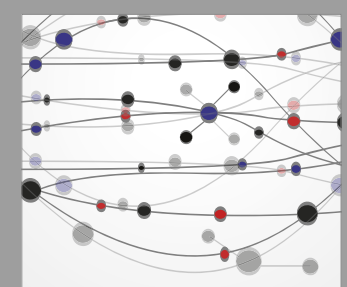

The Scientific World Journal
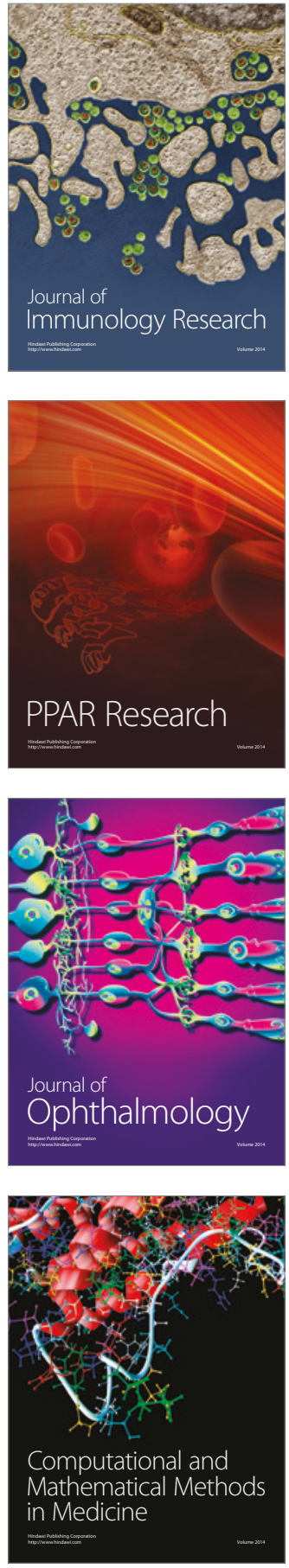

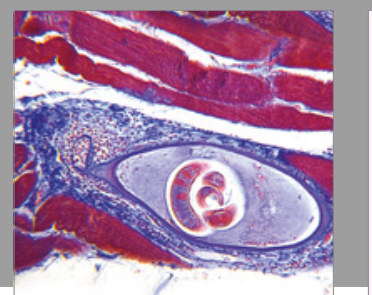

Gastroenterology Research and Practice
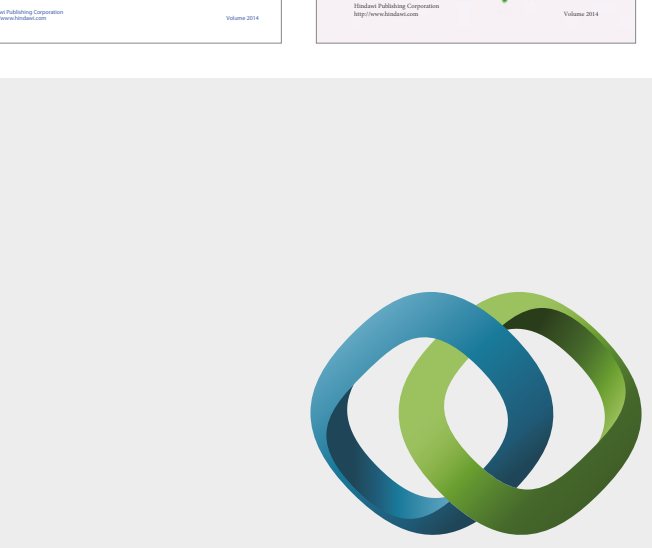

\section{Hindawi}

Submit your manuscripts at

https://www.hindawi.com
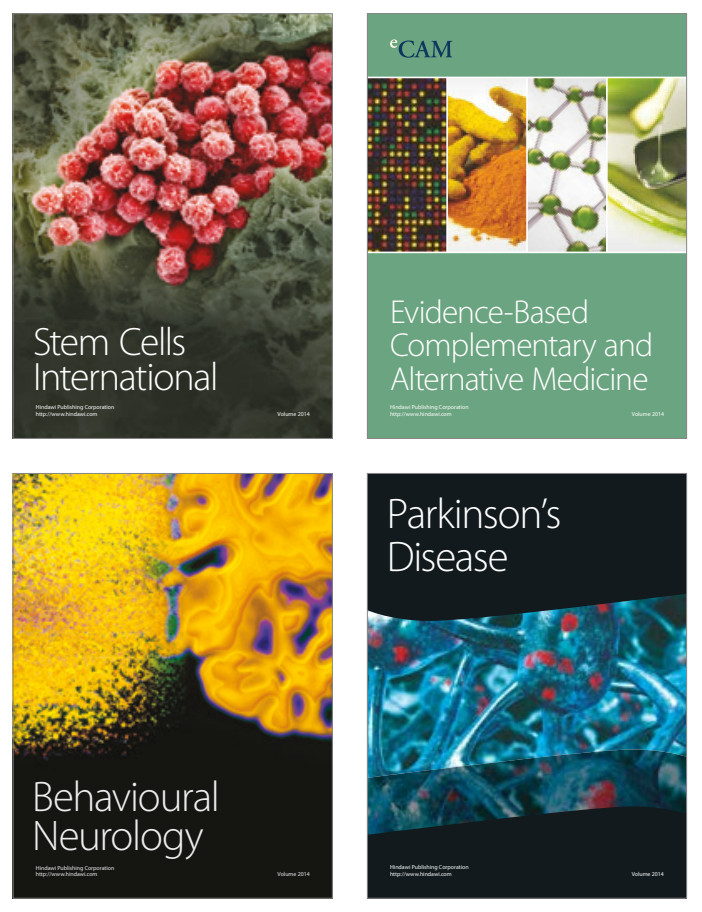
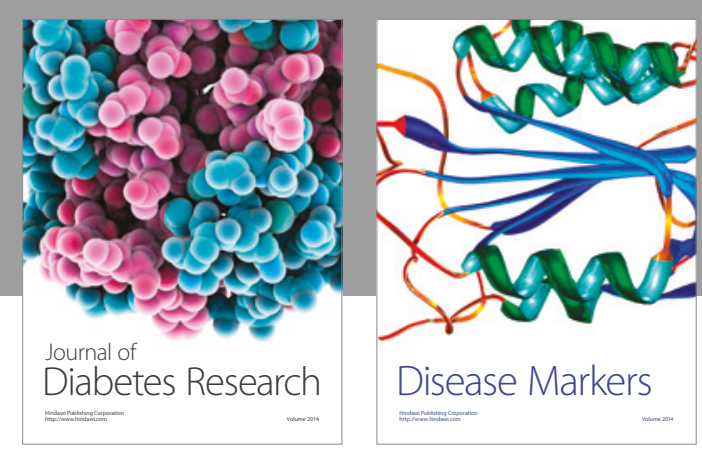

Disease Markers
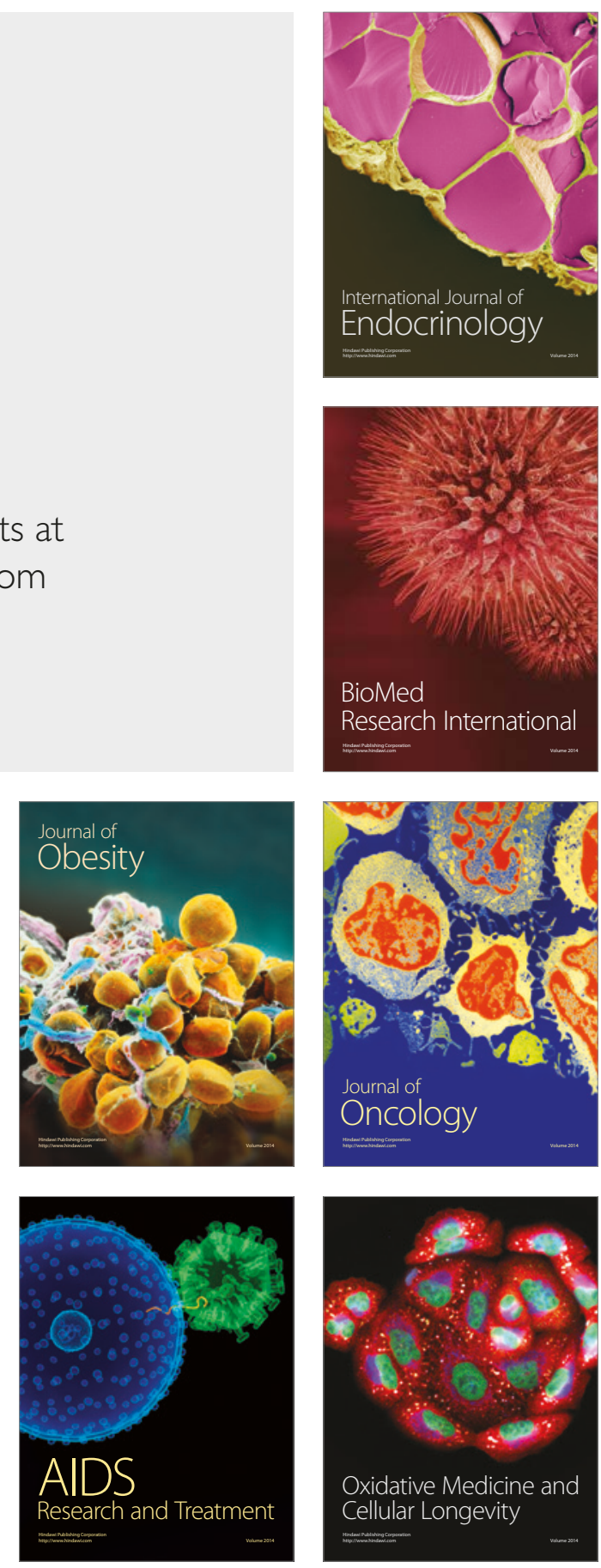